Türck ${ }^{1}$ stand damals im achtundvierzigsten Lebensjahre. Er hatte bereits auf neurologischem Gebiete Großes geleistet. Nun

Fig. 1.

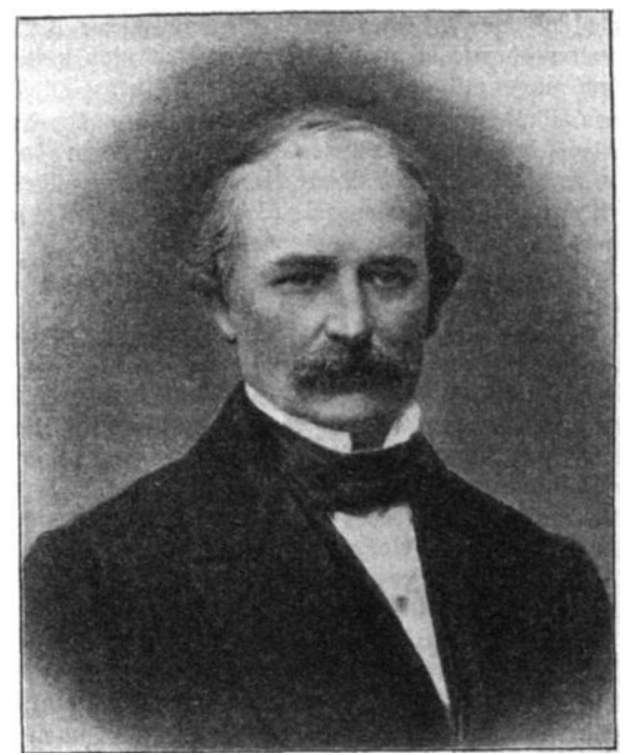

sollte er in einer ganz neuen Richtung bahnbrechend wirken. Das Schicksal wollte, daß $\mathrm{ihm}$, schon bevor seine Studien ausgereift und seine Resultate der breiten Oeffentlichkeit übergeben waren, ein sehr energischer Schrittmacher in Joh. Nepomuk Czermak dem damaligen Physiologen in Budapest, erstand. In edlem Wetteifer setzten beide Männer ihre ganzen Kräfte für die neue Sache

\title{
Zur Türck-Czermak-Feier in Wien, 21. bis 25. April 1908.
}

Von Gustav Killian in Freiburg im Breisgau.

Im Frühling des Jahres 1858 erfuhr die medizinische Welt von der Erfindung des Kehlkopfspiegels. Selten hat eine so unscheinbare Tatsache so weittragende Folgen gehabt. Heute, nach 50 Jahren, blicken wir voll Genugtuung und Stolz auf den stattlichen Bau, der auf diesem kleinen Grundstein errichtet wurde. Eine neue, interessante und vielgestaltige Wissenschaft ist erstanden, von der ein großer Segen für die leidende Menschheit ausgeht.

Solange die Medizin besteht, hat man auf laryngologischem Gebiet Erfahrungen gesammelt. Ein erhöhtes Interesse aber zeigte sich in der ersten Hälfte des neunzehnten Jahrhunderts, als B ozin $i$ mit seiner Schrift über die Lichtleiter hervortrat und eine Reihe von Männern wie Senn, Babington, Bennati, Baumes, Liston, Avery u. a. sich das Kehlkopfinnere mittels Spiegels sichtbar zu machen suchten. Auch von seiten der Anatomen (Henle), Physiologen (Johannes Müller) und besonders der pathologischen Anatomen (Cruveillier, Rokitansky) fand damals der Larynx mehr Beachtung. Zugleich bemühten sich die inneren Kliniker, auf dem Wege der Diagnostik und Therapie weiter zu kommen (Albers, Ryland, Trousseaux und Belloc, Rühle, Friedreich). So kam es, daß Ende der fünfziger Jahre die Kehlkopfkrankheiten schon zu den bedeutungsvollsten in der ganzen Pathologie gerechnet wurden. ${ }^{3}$ )

Aber was will das alles bedeuten gegen heute! Wieviel unlösbare Rätsel gab dąmals die alltägliche Praxis dem Arzte auf! Wie eng begrenzt war sein therapeutisches Können! Das meiste, was die Toten lehrten, konnte für die Lebenden nicht ausgenutzt werden. - Es mußte Wandel geschaffen werden. Die leidende Menschheit verlangte danach. Die endgültige Lösung des Problems der Laryngoskopie lag geradezu in der Luft. Schon hatte Manuel Garcia - den wir vor kurzem als über Hundertjahrigen zu Grabe getragen haben - 1854 an seinem eigenen Kehlkopf mit der Spiegeluntersuchung Erfolg gehabt. Da gelang es Ludwig Tü rck in Wien im Sommer 185 ? zum erstenmal, das Kehlkopfinnere eines kranken Menschen mit dem Spiegel zu sehen und zu demonstrieren. Damit war für die Medizin endlich ein langersehntes Ziel erreicht.

Fig. 2.

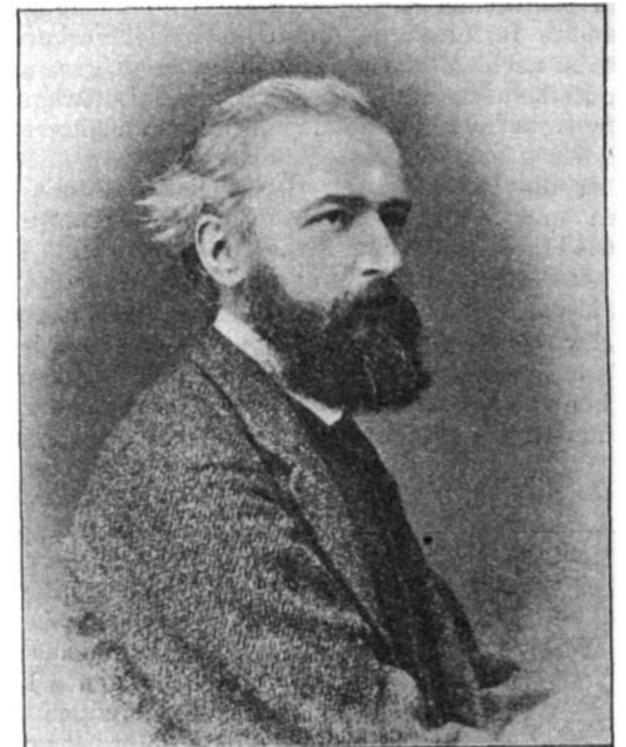

ein. Ozermak nutzte seine Erfahrungen auf dem Gebiete der Optik aus, machte sich durch Einführung der künstlichen Beleuchtung unabhängig vom Sonnenlicht und bemühte sich besonders um die Technik, während er das Klinische mehr zur Illustration benutzte. Türcks Ziel war dagegen, die Methode individuell auszugestalten, dem kranken Menschen möglichst gerecht zu werden und dessen Leiden mit den neuen Hilfsmitteln zu erforschen. Czermak lehrte uns die Luftrobhre von oben und von der Tracheotomiewunde aus mit dem Spiegel untersuchen, erschlob uns unter Anwendung desselben Prinzips das Innere des Nasenrachens und die hinteren Abschnitte der Nasenhöhle und hatte zuerst den mutigen Gedanken, die Spiegeltechnik lokal therapeutisch zu verwerten. Auch soll ihm nicht vergessen werden, daß er im In- und Ausland persönlich für die neue Sache Propaganda machte und ihr so eine rasche Verbreitung unter den Aerzten sicherte. Unter- 
dessen hatte Türck mit Riesenfleiß an dem Aufbau einer durch die Laryngoskopie umgestalteten Lehre von den Krapkheiten des Kehlkopfs und der Luftröhre gearbeitet und trat im Jahre 1866 mit einem klassischen, für alle Zeiten grundlegenden Werke hervor. Damit fand seine Lebensarbeit einen würdigen Abschluß. Schon bald danach (1868) raffte ihn der Tod nach kurzer Krankheit hinweg. Und seltsamerweise, auch $\mathrm{Ozermak}$ hat sich seit dieser Zeit nicht mehr auf laryngologischem Gebiet betätigt.

Mittlerweile aber war die Saat, die beide gesäet hatten, aller Orten mächtig aufgegangen. Leute, wie Störk, Semeleder, Gerhardt, Lewin, Rauchfuss, Voltolini, Viktor v. Bruns, Waldenburg. Traube, Tobold, Mackenzie, Elsberg, Mandl, Moura-Bourillou, Fauvel, Oertel, Gottstein, Wilhelm Meyer Navratil, Löri, Krishaber, v. Ziemssen, Schnitzler, Leopold v. Schrötter und spater Rossbach, Riegel, Baumler, Solis Cohen, Massei, Bschorner, Hopmann, S vielen anderen widmeten sich wissenschaftlich und praktisch der neuen Lehre. Fast alle waren noch jung an Jahren, als sie zum Spiegel griffen, und die Wenigen aus der mittleren Höhe des Lebens besaßen noch ihre jugendliche Begeisterungsfahigkeit. Es muß dies betont werden; ist doch die Jugend vorurteilsfreier und dem neuen mehr zugetan und fällt ihr doch die Erlernung neuer, insbesondere manueller Techniken leichter. Eine gewisse Geschicklichkeit war aber in der Laryngologie gerade wie in der Ophthalmologie unentbehrlich.

Jeder von den Genannten griff in seiner Weise die neue Materie an und baute aus seinen Beobachtungen auf. Die Folge war die raschePublikation einer außerordentlich umfangreichen und vielgestaltigen Kasuistik. Im Mittelpunkt des Interesses stand nach wie vor der Kehlkopf. Seine samtlichen Erkrankungen konnten jetzt am Le: benden genau studiert werden. Die bei den einzelnen Larynxaffektionen erzielten Fortschritte brauche ich hier nicht näher aufzufuhren. Besonders gewann die Lehre von den Neubildungen. Der komplizierte Muskel- und Nervenapparat des Kehlkopfes reizte zum Studium seiner normalen und gestörten Funktionen (Gerhardt). Die Krankheiten der Luftröhre lockten besonders $L$. v. Schrötter. Voltolini bearbeitete mit Vorliebe den Nasopharynx, in welchem Wilhelm Meyer schon im Jahre 1868 die adenoiden Vegetationen entdeckte.

An den sämtlichen Hilfsmitteln zur Untersuchung und an der Methodik mußte im Laufe der Jahre noch viel verbessert werden. Das Bedürfnis nach Milderung der mannigfaltigen und oft sehr energischen Reflexe im Bereiche der oberen Luftwege war groß, konnte aber trotz aller Bemühungen nur in mäßigem Grade befriedigt werden.

Auch an die Untersuchung der Speiseröhre mit Dilatationsinstrumenten und Spiegeln wagte man sich heran (Semeleder Störk, Voltolini), allerdings ohne rechten Erfolg. Dabei wußte man nicht, daß schon 1868 Kussmaul auf dem erst später endgiiltig gewählten, direkten Wege zum Ziel gekommen war.

Das Schwergewicht lag überall auf der Therapie. Ihrer rationellen Verbesserung galt viel Denken und Streben und Beobachten. Schon die bis dahin geübten allgemeinen Behandlungsmethoden mußten wesentlich modifiziert werden. Die lokalen aber

Fig. 3.

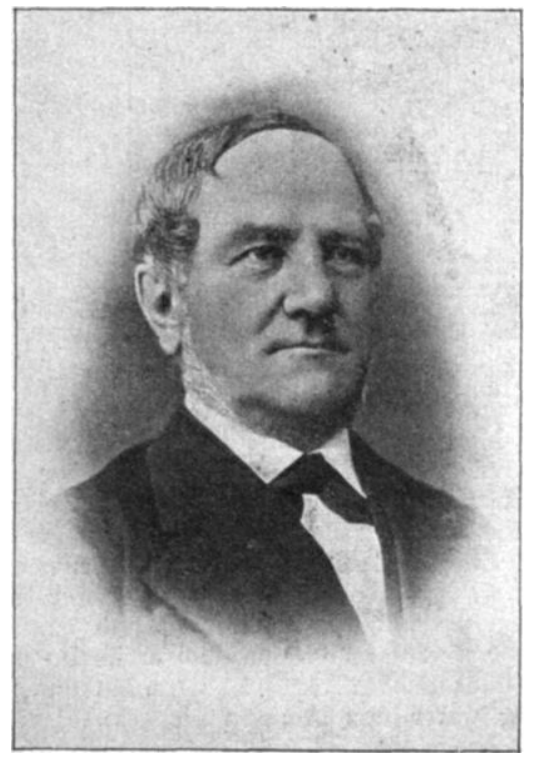

nen und Instrumente einleitet: „Operationen in der Kehlkopfhöhle des lebenden Menschen, in einem kleinen engen Raume, der iiber dies von sehr empfindlichen und in fast beständiger Bewegung begriffenen Wandungen begrenzt ist, von Wandungen, die sich bei der leisesten Beriihrung meist bis zur Aufhebung des Höhlenraums zllsammenziehen unter gleichzeitigem Eintritte heftiger Husten-, Würg oder Brechbewegungen, in einem Raume, ill welchem die erforderlichen Instrumente nur auf einen längeren, rechtrinklig geknickten Wege eingeführt werden können und wo dic Bewegungen dieser Instrumente, sowie das Operationsfeld selbst vou dem Auge des Arztes nur in dem Spiegelbilde überwacht werden können - Operationen an solcher Stelle gehören ohne allell Wider. spruch zu den schwierigsten in dem groben Gebieteder Chirurgie." End doch behielt dieser Satz ein Vierteljahrhundert lang seine Geltung. Und doch mußten unsere alten Laryngologen so lange Zeit ihr hohes Maß von Geschicklichkeit, Uebung, Geduld, Nachsicht und Erfahrung alltaglich ins Feld führen, um all der Schwierigkeiten Herr zı werden. Wie leicht werden derartige Dinge vergessen!

Die in der Laryngologie erzielten Fortschritte gaben auch der übrigen Medizin neue Anregungen. Besonders gewann die innere Klinik, aber auch die Chirurgie wurde beeinflulist. Das Jahr 1873 brachte nach Czernys Vorversuchen die erste Entfernung des ganzen Kehlkopfes beim lebenden Menschen durch Billroth.

$W$ as diese erste Periode alles geleistet hat, ist aus der 1876 erschienenen ersten Auflage des v. Ziemssenschen Werkes der speziellen Pathologie und Therapie leicht zu ersehen. Mit Recht sagt hier der Autor: "Eine so rasche und glänzende Entwicklung, wie sie die Kehlkopflehre während eines Zeitraums von kaum zwanzig Jahren erfahren hat, möchte in der Geschichte der Medizin schwerlich ihres gleichen haben".

\section{Zweite Periode, 1876 - 1884.}

Dieser ersten rein laryngologischen Blitezeit folgte eine zweite, bis zu dem Cocainjahr 1884 reichende, in welcher die $\mathrm{Rl}_{1} \mathrm{i}-$ nologie neu erweckt nnd der Laryngologie angegliedert wurde. Die Anfänge dieser Entwicklungsphase reichen zwar schon bis in die erste Hälfte der Siebziger Jahre zurück, deutlich in Erscheinung aber trat sie erst im Jahre 1876 , als die ersten Bearbeitungen der Nasenkrankheiten von Bernhard Fränkel und von Michel erschienen.

Wesentlich kürzer wie die vorige Periode, hat sie doch $\mathrm{Her}$ vorragendes geleistet, obwohl von den alten Laryngologen schon manche ausgeschieden und neue Namen in den Vordergrund ge treten waren: Bernhard Fränel, Spencer Watson, Michel, Scheff, Böker, Jelenffy, Arthur Hartmann, Iefferts, Jurasz, Moritz Schmidt, Bresgen, Zaufal, Pieniazek, Chiari, Seifert, Krause, Garel, Schiffers, Eletscher Ingals, Bosworth, Gougenheim, Bayer, Moldenhauer, Hack, Schäfer u. a.

Der weitere Ausbau der Laryngologie wurde inımer schwieriger Das Neue fiel dem Beobachter nicht mehr wie früher geradezu in den Schoß. Es galt vielmehr jetzt, das ganze Gebiet wissenschaftlich zu vertiefen. Obwohl sich diesem Bestreben große Schwierigkeiten entgegenstellten, Taboratorien und wissenschaftliche Hilfsmittel nicht hinreichend benutzt werden konnten und die Polikliniken nur über dürftige Einrichtungen verfügten, wurde docl sehr eifrig gearbeitet. Man warf sich auf die Entwicklungsgeschichte die vergleichende Anatomie und besonders auf die Physiologie (Schech, Krause, Oertel u. a.). Schon wurde die zentrale Repräsentation des Larynx in Angriff genommen (H. Krause 1883).

Klinisch kamen außer der Technik und lokalen Therapie besonders die Fremdkörper und die Stenosen in Kehlkopf- und Luft röhre in Betracht (L. v. Schrötter). Im Jahre 1892 brachte diè Entdeckung des Tuberkelbacillus durch Koch die Klarung des Begriffes der Tuborkulose und damit zugleich ihrer verschiedenen Formen im Bereich der Luftwege und oberen Speisewege.

Große Anfmerksamkeit fanden die Krankheiten des Rachens und des Nasenrachens. Das Krankheitsbild der adenoiden Vegetationen veranlaßte hohe Literaturwellen. Auclı die Beziehungen der Laryngologie zur Gesamtmedizin fanden eine eingehende Bearbeitung (Löri).

Was nun die Rhinologie angeht, so war sie bis zum Anfang dieser Periode, wie von alters her, immer noch das Stiefkind der Medizin geblieben. Selbst die pathologische Anatomie hatte hier fast ganzlich versagt. Die Krankheiten der Nase und ihrer Nebenhöhlen wurden bis dato vorwiegend von den Chirurgen beobachtet und behandelt und in deren Lehrbiichern besprochen. Ueber eine gewisse Grenze kam man nicht hinaus. Die schon seit dem Mittelalter her bekannte vordere Rhinoskopie mit Specula (Guy de Chauliac 1363), für welche - abgesehen von der Beleuchtung mit Sonnenstrahlen durch ein Loch im Fensterladen. - Gul. Cesare Aranzi 
(Bolognia 1587) schon die künstliche Beleuchtung mit Kerze und Schusterkugel ausgedacht hatte, wurde kaum geübt. Alles muBte neu anfgebaut werden, ja man machte sich die Arbeit dadurch noch besonders schwer, daß man die ältere Literatur nicht hinreichend studierte. Von einigen kleineren Arbeiten abgesehen, zeigten sich zuerst in dem Buche von Michel bemerkenswerte Fortschritte und selbst der Aufang einer neuen Lehre von den Erkrankungen der Nebenhöhlen der Nase.

Nachdem einmal das Interesse auf dieses neue Gebiet hingelenkt war, wetteiferten Laryngologen und Otologen in der Bearbeitung desselben. Der Stirnspiegel, die verbesserten Lichtquellen und Specula, die fleißige Benutzung der Sonde gestatteten in der Tat eine wesentlich bessere Orientierung in dem komplizierten Innern der Nasenhöhlen, als sie früher möglich gewesen war. Zur rechten Zeit schuf $Z$ uckerkandl verbesserte anatomische und pathologisch - anatomische Grundlagen. Es zeigte sich bald, dal sich in den Nasenhöhlen eine ganze Reihe eigenartiger Vorgänge abspielt und daB innige Wechselbeziehungen zum Gehörorgan und zu den tieferen Abschnitten der Luftwege bestehen. Vieles blieb allerdings immer noch rätselhaft, weil man die Nebenhöhlenaffek tionen meist noch übersah. Die lokale Therapie verlangte auch auf diesem Gebiet ein eingehendes Studium und ein neues und eigenartiges Instrumentarium. Fin souveränes Hilfsmittel war in jener Keit nach Voltolinis Vorgang die Galvanokaustik.

Die gesamten Errungenschaften der ersten und zweiten Periode unserer Wissenschaft sind in einer Reihe von Lehrbüchern fest gelegt, am umfassendsten in dem zweibandigen großen Werke von Mackenzie und Semon. Dort sind auch schon die Krankheiten der Speiseröhre hinzugenommen. (Ihre Darstellung ist jedoch noch nicht von der $188 \mathrm{~J}$ erfundenen v. Mikuliczschen Oesophagoskopie beeinflußt.) Hier erscheint also unser Fach schon so kompakt, wie wir es auch heutzutage mit aller Konsequenz vertreten.

Als ein besonders bedeutungsvolles Ereignis, welches mit dem Ende der zweiten Periode zusammenfällt, muß ich die Gründung des internationalen Centralblattes für Laryngologie durch Felix Semon bezeichnen, welches von jetzt ab unsere umfangliche und weit zerstreute Literatur übersichtlich zusammenfaßte und die wissenschaftliche Arbeit außerordentlich erleichterte und förderte.

Dritte Periode, 1884-1895.

Im Jahre 1884 machte uns Jelinek in Wien mit der anästhesierenden $\mathrm{W}^{\prime}$ irkung des Cocains auf die Rachen- und Kehlkopfschleimhaut bekannt. Das war eine befreiende Tat! Wie oft hatten wir uns ein solches Zaubermittel gewünscht! Wie oft gezweifelt ob es je gefunden werden könne. Nun war es da, und ein anBerordentlicher Segen ging von ihm aus.

Reflexe konnten gedämpft und beseitigt, Schmerzen konnte vorgebeugt werden. Die Untersuchungen wurden ungemein erleichtert; sie ließen sich häufiger und selbst unter den schwierig sten Bedingungen mit Erfolg ausführen. Wesentliche Verbesserungen der alten Methoden wurden nun möglich. Ja auf der Basis der lokalen Anästhesie entstanden ganz neue und wichtige Unter suchungsarten.

Davon hatten die gesamten oberen Luft- und Speisewege Nutzen, nicht zum geringsten die Nasenhöhlen, deren Schleimhaut durch das Cocain nicht allein unempfindlich gemacht, sondern auch zur Abschwellung gebracht wurde.

An jede Stelle in unserem ganzen, großen Gebiete konnten wir unter dem Schutze des neuen Mittels jetzt mit Instrumenten leicht hingelangen und die notwendigen Prïfungen und probatorischen Maßnahmen vornehmen. Die ganze Diagnostik erhob sich rasch weit über das frühere Niveau, nicht minder die Therapie. Die vielen kleinen und großen unerläßlichen Eingriffe schmerzlos gestalten zu können, das war für Arzt sowohl wie für Patient ein fast idealer Zustand. Jetzt schenkte uns endlich der Patient die nötige Zeit zu allem. Auf die Schnelligkeit des Eingreifens kam es nicht mehr so an; es konnte viel exakter und gründlicher behandelt wer den. Auch lie 3 sich in einer einzelnen Sitzung viel mehr erreichen. Wo man frijher etappenweise vorgehen mußte und mit all den Ge fahren intercurrenter Entzïndungen zu rechnen hatte, gab es jetzt glatte Fingriffe mit primären Heilungen. Es ist nicht zuviel gesagt, wenn ich behaupte, daß dies Wundermittel in der LaryngoRhinologie dieselbe Stufe einnimmt wie die allgemeinen Narcotica in der Chirurgie.

Auch in der Laryngologie kam es rasch zu einer vollständigen Umwälzung des Faches. Ja sogar seine ärztliche Vertretung änderte sich. Jene kïnstlerische Geschicklichkeit der alten Meister blieb nicht mehr conditio sine qua non für die Wahl der Laryngo-Rhinologie zum speziellen Beruf. Auch gewöhnliche Sterbliche mit einigermaßen gewandter Hand konnten sich jetzt in unser Arbeitsgebiet einleben lind mit Uebung und Geduld der Technik Herr werden. Daher nahm die Zahl der Spezialisten rasch zu, und die Segnıngen unseres
Wirkens kamen von jetzt ab den breitesten Schichten des Volkes zugute.

Mit der Zunahme der Vertreter engte sich der praktische Wirkungskreis des einzelnen ein, Er wurde, was früher nicht in dem $\mathrm{Maße}$ der Fall gewesen war, genötigt, die gesamten oberen Luftund Speisewege zu seinem Arbeitsfeld zu machen, sein Wissen möglichst zu vertiefen und seine Leistungen auf das höchste Maß zu bringen. Der Kampf ums Dasein trieb ihn jetzt vorwärts.

Diesem Umstande ist es auch zuzuschreiben, daß die LaryngoRhinologen von jetzt ab die Otologie und viele Otologen die Laryngo-Rhinologie hinzunahmen. So haben die äußeren Umstände drei Gebiete zur Verschmelzung gebracht, welche ihrem inneren Wesen nach klinisch durchaus zusammengehören.

Es ist mir kaum noch möglich, die Namen der Männer alle zil nennen, die sich von jetzt $a b$ hervortaten und Bausteine herbeischleppten, selbst wenn ich nur den ersten Abschnitt des Zeitalters der lokalen Schleimhaut-Anästhesie bis 1895 ins Auge fasse. An bemerkenswerten Fortschritten dieser Zeit auf laryngologischem Gebiet nenne ich im einzelnen die Untersuchung des Kehlkopfes und der Luftröhre bei vorgebengtem Kopfe, wodurch die Hinterfläche der Kehlkopfhöhle und schwierigere Trachealfälle der Untersnchung zugänglich wurden; ferner die chirurgische Behandlung der Larynx-Tuberkulose (Moritz Schmidt, Heryng), unterstützt durch die Anwendung der Milchsäure (H. Krause); die Behandlung akuter und chronischer Larynxstenosen mit $O^{\prime} \mathrm{D}$ wy erscher Intubation; die Behringsche Heilserumbehandlung der Diphtherie; die häufigere Verwertung der Laryngofissur bei hochgradigen Narbenstenosen und bösartigen Neubildungen des Kehlkopfes (Pieniazek). Man erforschte das Sklerom und unterzog die Posticuslähmung erneuter gründlicher Bearbeitung, stritt sich heiß um das Rosenbach-Semonsche Gesetz und suchte eine breitere, wissenschaftliche Basis für die peripherische und zentrale Innervation des Kehlkopfes (Exner, Onodi, Grabower). Zugleich erreichte die Laryngologie einen Höhepunkt durch die Arbeiten von Semon und Horsley über die Gehirnzentren des Kehlkopfes.

Noch viel lebhafter ging es auf rhinologischem Gebiet zu. Hacks enthusiastische Behandlung der Reflexneurosen erregte geradezu eine Revolution. Der Cauter witete. Daneben schufen Arthur Hartmann, Moldenhauer u. a. ruhigere, vorurteilsfreiere Arbait. Man lernte in der Rhinitis fibrinosa (H artmann) eine abgeschwächte Form der Nasen-Diphtherie kennen; Nasen-Syphilis und Nasen-Lupus wurden genauer studiert. Im Nasenrachen entstand über die Bursa pharyngea und ihre Erkrankungen (Thornwald) eine ganze Llteratur; daneben blühten die verschiedenen Operationsmethoden der hypertrophischen Rachentonsille. Schwabach und G. Killian gingen diesen Dingen anatomisch, vergleichend und entwicklungsgeschichtlich zu Leib.

Vor allem aber, und das ist eine Hauptsignatur dieser Epoche, feierte die Lehre von den Erkrankungen der Nebenhöhlen der Nase ihre Auferstehung. Hier hat $\mathrm{Ziem}$ das große Verdienst, außerordentlich anregend gewirkt zu haben (schon seit 1880). Die Rhinoscopia anterior und posterior, durch das Cocain unterstützt, ermöglichte in ausgezeichneter Weise die intranasale Untersuchung der Nebenhöhlen. Zunächst wurde die Kieferhöhle mit intranasalen Sondierungen, Kanülen-Spülungen und Probepunktionen erschlossen (Joh. Killian, Hartmann, M. Schmidt, Krause etc.). Dazu kam die viel diskutierte Durchleuchtung der Höhle (Heryng). Die Lehre von den Erkrankungen der Stirnhöhlen, Siebbeinzellen und Keilbeinhöhlen wuchs auf der Basis der großen Influenza-Epidemien rasch empor. 1887 hatte $J$ urasz seine Sondierung des Sinus frontalis angegeben; für die Erkrankungen der Siebbeinzellen und Keilbeinhöhlen brachten Berger und Tyrmann die literarische Basis. Auch wirkte Scha effer (Bremen) mit seiner Löffelsonde hior bahnbrechend und von Vorurteilen befreiend, und Jansen zog spater die großchirurgischen Konsequenzen daraus, nachdem die alte Kieferund Stirnhöhlen-Chirurgie wieder aufgelebt war. Fur die Verfeinerung der Diagnosestellung und besonders ftir die Differentialdiagnose der Erkrankungen der einzelnen Nebenhöhlenräume arbeiteten Lichtwitz, Luc, G. Killian, $\mathrm{H}$ ajek und viele andere. Dazu brachte Gruinwald mit seinem geistreichen Buche iber die Naseneiterungen in die ganze Nebenhöhlenlehre einen großen Stil Auch die pathologische Anatomie dieser Höhlen fand endlich mehr Beriicksichtigung (Harke, E. Fränkel u. a.)

Durch die Aufklärungen tiber die Erkrankungen der Nasennebenhöhlen gewann die Lehre von den Krankheiten "der Haupt höhlen außerordentlich; denn sie wurde von vielen. Fehlern befreit.

Die bessere Beurteilung der Bedeutung von Nasen- und Mundatmung (Bloch), angebahnt durch die Lehre von den adenoiden Vegetationen und ihren Folgezuständen griff damals ebenfalls mehr Platz. Die bessere Würdigung der häufigen mechanischen Störungen der Nasenatmung und deren Folgen für die Erkrankungen des ganzen Pharyngo-, Laryngo-, Tracheal- und Bronchial-Gebietes 
niitzte dem Ganzen ungemein und bahnte eine neve, rationellere, den Ursachen mehr Rechnung tragende Therapie an, welche viele positive Heilerfolge herbeifïhrte.

Gegen Ende der dritten Periode wurde in den meisten laryngorhinologischen Studierstuben ungemein fleiligig gearbeitet, denn es galt, die gesamte Weltliteratır durchzıwühlen und alles, was für das Fach nur irgend von Bedentung war, zusammenzuschleppen, um ein großes Werk zustande zu bringen, welches aus dem gesamten Wissen abdestilliert war. Unter der Redaktion von P. Heymann wuchs es zı fünf stattlichen Bänden lıeran und wurde zu einem unentbehilichen Nachschlagebuch für alle Zeiten.

\section{Vierte Periode. 1895 bis heute.}

$M$ an hätte glauben sollen, daß jetzt eine ruhigere Zeit beginnen würde; aber es kam ganz anders. Die direkten Untersuchungsmethoden erschienen und fachten neues Leben an. Zuerst die Kirsteinsche direkte Laryngoskopie, dann meine direkte TracheoBronchoskopie. Von der Tracheotomiewunde aus ausgefiihrt, ergänzte sie die ältere Pieniazeksche untere Tracheoskopie.

Bei der Verwendung lokaler und allgemeiner Anästhetica erwiesen sich diese Methoden bald für jedes Alter bis zum Säugling hinab anwendbar. Was früher mit dem Spiegel nicht bezwungen werden konnte, wurde jetzt der Betrachtung zugänglich. Damit erst errangen wir die ranze Herrschaft ïber unser großes und schwieriges Gebiet. Zugleich befähigten uns die neneu Methoden, genauer und besser zul sehen. Die Bronchoskopie eroberte uns ein nenes Feld, sie machte nuserem Auge die Bronchialverzweigungen zugänglich und ermöglichte uns, auf einem nenen diagnostischen Wege den Affektioneu des Lungengewebes näherzntreten. Die Grenzgebiete mit der innereu Medizin und Chirurgie im Bereiche des Halses und der Brust dehnten sich aus, soweit Lage, Form, Gewebs- und Inlaaltsveränderungen der Luftröhre uud der Bronchien in Betracht kamen.

Unübersehbar war der therapeutische Gewinu. Durch unsere Röhrenspatel und unsere Röhren hindurch wurden die erkrankten Bezirke bis tief in die Lunge fiur die verschiedenartigsten Instrumente zugänglich. Am meisten hatte bisher die Lehre von den Fremdkörpern Vorteil davon. In zahlreichen F'ällen hat man Freındkörper nicht allein von der Tracheotomiewunde aus, sondern auch auf natürlichem Wege aus den Tiefen der Lunge mit Gliick entfernt. Ihren Höhepunkt erreichte diese Technik bis jetzt durch unseren gerade publizierten Fall, in dem ein Fremdkörper, der fïn! Jahre in der Lunge gesteckt und sich fast vollständig hinter einer derben Bindegewebsstenose verborgen hatte, nach allmählicher Dilatation der Stenose glücklich entfernt wurde und bei dem in der Nachbehandlung zum ersten Male die Bronchus-Intubation mit freiem Metalltubus zur Anwendung kam. (Killian und Brünings, Zeitschrift für Ohrenheilkunde und fïr die Krankheiten der Luftwege 1908 , Bd. 55, H. 4.)

Aber auch bei gutartigen Neubildungen im Larynx und in den tieferen Luftwegen ist man mit bestem Erfolg operativ vorgegangen. Wer hätte je geahnt, daßj es einmal gelingen könnte, ein im Bronchus gewachsenes, polypöses Ecchondrom anf natürlichen Wege dauernd zu beseitigen (v. Eicken)! Bei den bösartigen Geschwülsten haben uns wenigstens die tracheobronchoskopischen Probeexzisionen in der Beurteilung der Fälle wesentlich gefördert.

In analoger Weise wie in den Luftwegen, ja sogar mit demselben Instrumentarium (das nellerdings durch Brünings eine so außerordentliche Verbesserung erfahren hat) konnte diagnostisch und therapeutisch in der Speiseröhre vorgegangen werden. Die Laryngologie hat sich daher endgültig in dem Gebiete der Speiseröhrenerkrankungen eingenistet, indem sie sich ihre großen technischen Erfahrungen im Bereiche des Halses zunutze machte. Schon hat sie bemerkenswerte positive Arbeit auf diesem Gebiete geleistet. Sie bleibt dabei eingedenk der Tatsache, da/3 die direkte Oesophagoskopie anf chirurgischem und intern-medizinischem Boden gewachsen ist (Kussmaul, v. Mikulicz, v. Hacker, Rosenheim). Ich versäume nicht, zu erwähnen, daß anclı die Gastroskopie in verbesserter Form in den Händen der Laryngologen wieder aufzuleben scheint (Chevalier, Jackson).

Fast in dieselbe Zeit fallen die grolsen Fortschritte, welche uns die Röntgenuntersuchungen gebracht haben. Diese bestätigen in willkommener Weise, was wir mit direkten und indirekten Methoden im Bereich des Halses nnd der Brust feststellen, ergänzen die Lücken unserer Beobachtungen und fördern in ansgezeichneter Weise die Diagnostik und Therapie der Nasennebenhöhlenerkrankungen. Zeichnet uns doch die Röntgenphotographie geradezı diagrammatisch die ganzen Höhlensysteme auf, erkennen wir doch oft unmittelbar ans dem Bilde, was gesund und was krunk ist.

Als weiteren wesentlichen Fortschritt bezeichne ich die Tatsache, dals wir im kleinen und groljen chirurgisch denken und handeln gelernt haben. Dadurch wurden oft Erfolge erzielt, wo die frühere
Therapie ungenïgend und ohnmächtig gewesen war. Auch die gewissenhafte Verwertung der`Asepsis, ferner die Schleichschen lujektionen, nicht allein außen, sondern anch im Inuern der Hollräume ausgeführt, die Paraffininjektionen und viele andere $\mathrm{Er}$ rungenschaften der modernen Chirurgie haben uns großen Nutzen gebracht.

Dabei sind wir von der inneren Medizin nicht abgewichen und haben nach wie vor reiche Anregungen und therapeutische Vorteile aus ihr geschöpft. Die neueren diagnostischen Reaktionsmethoden, die neu entdeckten Krankheitserreger, die Serumtherapie, die Sandtoriumsbehandlung der 'Tuberkulose seien nur al:i Beispiele genannt. Auch mit dem Gebiet der Otologie und namentlich der Ophthalmologie entwickelten sich nene, wichtige Wechselbeziehungen.

7u dieser allgemeinen Charakterisierung der vierten Periode kommt der Hinweis auf einige spexielle Erscheinungen. So die Glu cksche Methode der Larynxexstirpation, die Radikaloperationen der 'Tonsillen, die blutigen Operationen im Bereich der Muscheln (als Ersatz für den Cauter) und an der Nasenscheidewand (submuköse Fensterresektion). Hier sowie in der gesanten intranasal'n Therapie erwuchsen uns große Vorteile aus der Anwendung des Adrenalins, welches die Cocainwirkung verstärkte und die Blutungen meist so beschränkte, daß wir wie am Phantom operierten konnten.

Bei alledem nahm die Lehre von clen Frkrankungen der Nasennebenhöhlen einen hohen Aufschwung. Das genaue Studium der Anatomie und besonders der Topographie (G. Killian, Onodi, Logan, Turner 1. a.) gab der Diagnostik eine exakte Basis. Die Stirnhöhlendurchleuchtung (Vohsen), die Rhinoscopia media, die verschiedenen Formen der Probeexplorationen zusammen mit der schou erwähnten Röutgenphotographie brachten groß3e Klarheit und Sicherheit. Dazı gewannen wir eine genauere Kenntnis der fortschreitenden entziindlichen Affektionen uud ihrer inannigfaltigen und gefährlichen Komplikationen in nahen und entfernteı Organen. $\mathrm{Zn}$ gleich funden wir die Mittel und Wege durch allgemeine Behand. lung, durcli intra- und extranasale chirurgische Therapie, die $\mathrm{Er}$ krankungen der Nasennebenhöhlen und ihre Komplikationen wirksam zu bekämpfen. Die Lucsche Radikaloperation der Kieferhöhle und die Killiansche Radikaloperation der Stirnhöhle seien hier besonders erwähnt.

Das historische Bild wäre nicht vollständig ohne den Hinwois, daß die Lehre von den Erkrankungen der Luftwege und der obureı Speisewege jetzt in allen Kulturländern durch zahlreiche Nerzte vertreten wird, die sich in vielen großen und kleinen Vereinigungen zusammengefunden haben und jährlich Kongresse veranstalten. In zahlreichen Lehrbüchern und Monographien ist das wissenschaftliche Naterial gesanmelt, und viele Zeitschrifteu dienen der Prom duktion des Tages. Aller Orten an den Universitäten und in großsen Verkehrszentren gibt es laryngo-rhinologische Poliklinikeu. Auch viele stationäre Kliniken sind entstanden. Ueberall wird eifrig gearbeitet und gelehrt.

So hat sich das Werk Türcks und Cuermaks nach einem halben Jahrhundert zu einer blübenden Wissenschaft entwickelt, die den anderen Spezialdisziplinen der Medizin wïrdig zur Seite steht.

Mit Freude und Genugtuung stellen wir dies heute fest und gedenken in tiefer Dankbarkeit der beiden Begrïnder unseres Faches. Ihr Vorbild leuchte uns voran bei unserer kiinftigen Arbeit harren unserer doch noch großse Aufgaben! 